\title{
Endpoint estimates from restricted rearrangement inequalities
}

María J. Carro and Joaquim Martín

\begin{abstract}
Let $T$ be a sublinear operator such that $(T f)^{*}(t) \leq h\left(t,\|f\|_{1}\right)$ for some positive function $h(t, s)$ and every function $f$ such that $\|f\|_{\infty} \leq 1$. Then, we show that $T$ can be extended continuously from a logarithmic type space into a weighted weak Lorentz space. This type of result is connected with the theory of restricted weak type extrapolation and extends a recent result of Arias-de-Reyna concerning the pointwise convergence of Fourier series to a much more general context.
\end{abstract}

\section{Introduction}

Let $S$ be the Carleson maximal operator (see [6])

$$
S f(x)=\sup _{n}\left|S_{n} f(x)\right|,
$$

where $S_{n} f(x)=\left(D_{n} * f\right)(x)$, being $D_{n}$ the Dirichlet kernel on $\mathbb{T}=\{z \in$ $\mathbb{C} ;|z|=1\}$ and $f \in L^{1}(\mathbb{T})$. Then, it was proved in [6] and [13] the following restricted weak-type estimate:

$$
\sup _{y>0} y \lambda_{S \chi_{A}}(y)^{1 / p} \leq \frac{C}{p-1}|A|^{1 / p},
$$

for every $1<p \leq 2$ and every measurable set $A$, with $C$ independent of $p$, where $\lambda_{g}(y)=|\{x \in \mathbb{T}:|g(x)|>y\}|$ is the distribution function of $g$ with respect to the Lebesgue measure. Using this estimate and Yano's extrapolation theorem (see [22] and [7]), one can easily see that

$$
S: L(\log L)^{2} \longrightarrow L^{1}
$$

is bounded.

2000 Mathematics Subject Classification: 46M35, 47A30.

Keywords: rearrangement inequality, real interpolation, Banach couples, extrapolation theory, Carleson's operator. 
However, in [18], this boundedness was improved by using that if we take the infimum in $p$ in the above inequality then, for every measurable set $E \subset \mathbb{T}$,

$$
\left(S \chi_{E}\right)^{*}(t) \preceq \frac{|E|}{t}\left(1+\log ^{+} \frac{t}{|E|}\right)
$$

$\left(g^{*}(t)=\inf \left\{s: \lambda_{g}(s) \leq t\right\}\right.$ is the decreasing rearrangement) and, proving that this estimates also holds by the so called special functions. Then, the boundedness of

$$
S: L \log L \log \log L \longrightarrow L^{1, \infty}
$$

was proved. Some years later, F. Soria in [20] improves the above extrapolation result by showing that

$$
S: B_{\varphi}^{*} \longrightarrow L^{1, \infty}
$$

is bounded with $\varphi(t)=t\left(1+\log ^{+} 1 / t\right)$ and $B_{\varphi}^{*}$ a block type space such that $L \log L \log \log L \subset B_{\varphi}^{*}$.

In 1996, Antonov (see [1]) proved the following lemma:

Lemma 1.1 (Antonov) Let $S^{N} f(x)=\sup _{0<n<N}\left|S_{n} f(x)\right|$. Then, for every $\varepsilon>0$, every $N \in \mathbb{N}$ and every $0 \leq f(x) \leq 1$, there exists a measurable set $F$ such that $|F|=\|f\|_{1}$ and $\left\|S^{N}\left(f-\chi_{F}\right)\right\|_{\infty} \leq \varepsilon$.

Using this result and the above estimate on characteristic functions one can conclude that

$$
(S f)^{*}(t) \preceq \frac{\|f\|_{1}}{t}\left(1+\log ^{+} \frac{t}{\|f\|_{1}}\right),
$$

for every $f \in L^{1}$ such that $\|f\|_{\infty} \leq 1$, and from it, Antonov proves that

$$
S: L \log L \log \log \log L \longrightarrow L^{1, \infty}
$$

is bounded. Quite recently, it has been proved by Arias-de-Reyna in [2] that

$$
S: Q A \longrightarrow L^{1, \infty}
$$

is bounded where $Q A$ is a rearrangement invariant space such that

$$
L \log L \log \log \log L \subset Q A \quad \text { and } \quad B_{\varphi}^{*} \subset Q A .
$$

Moreover, $Q A$ is strictly bigger than both spaces and therefore $Q A$ is, up to now, the biggest space where the pointwise convergence of the Fourier series is known to hold. 
Antonov's lemma has been extended in [19] to more general operators, namely to any maximal operator of the form

$$
T f(x)=\sup _{j}\left|K_{j} * f(x)\right|,
$$

where $K_{j} \in L^{1}$, and therefore, (1.2) holds for any operator $T$ of the above form such that $T$ satisfies (1.1). Examples of such operators are given in [19] in the setting of differentiation of integrals and the Halo conjecture.

In particular, (and this is the connection with the weak extrapolation theory, see [14] and [20]) if $T$ is an operator such that, for every $1<p \leq 2$,

$$
(T f)^{*}(t) t^{1 / p} \leq \frac{1}{(p-1)^{m}}\|f\|_{p}
$$

then, for every $f \in L^{1}$ such that $\|f\|_{\infty} \leq 1$,

$$
(T f)^{*}(t) \leq \frac{1}{(p-1)^{m}} \frac{\|f\|_{1}^{1 / p}}{t^{1 / p}}
$$

and taking the infimum in $p$, we conclude that

$$
(T f)^{*}(t) \preceq \frac{\|f\|_{1}}{t}\left(1+\log ^{+} \frac{t}{\|f\|_{1}}\right)^{m} .
$$

Our main purpose (see Theorem 3.1) is to show that if $T$ is a sublinear operator satisfying

$$
(T f)^{*}(t) \preceq h\left(t,\|f\|_{1}\right),
$$

for some positive function $h$ and every $\|f\|_{\infty} \leq 1$, then

$$
T: Q_{D} \longrightarrow M(R)
$$

is bounded, where $h(t, s) \leq D(s) R(t)$,

$$
Q_{D}=\left\{f ; f=\sum_{k} e_{k} f_{k},\left\|f_{k}\right\|_{\infty} \leq 1,\|f\|_{Q_{D}}<\infty\right\}
$$

with

$\|f\|_{Q_{D}}=\inf \left\{\sum_{k} e_{k} D\left(\left\|f_{k}\right\|_{1}\right)\left(1+\log \frac{1}{a_{k}}\right) ; \sum_{k} a_{k}=1, a_{k} \geq 0, f=\sum_{k} e_{k} f_{k}\right\}$,

and

$$
\|f\|_{M(R)}:=\sup _{t>0} \frac{f^{*}(t)}{R(t)}
$$

In particular, if

$$
D(s)=s\left(1+\log ^{+} \frac{1}{s}\right)
$$

and $T=S$, then our space $Q_{D}$ coincides with the space of Arias-de Reyna $Q A$. 
Our proof turns out to be very simple and is based in the following basic result (see [9]):

Lemma 1.2 (Basic result) Let $f=\sum_{n} f_{n}$ and let $c_{n}>0$ be such that $\sum_{n} c_{n}=1$. Then

$$
f^{*}(3 t) \leq \sum_{n}\left(f_{n}^{*}(t)+\frac{1}{t} \int_{c_{n} t}^{t} f_{n}^{*}(s) d s\right) .
$$

From it, the main result of this paper, which covers as a particular case the result of Arias-de-Reyna, can be immediately obtained.

The point now is that the space $Q_{D}$ is difficult to handle and, therefore, it is convenient for the applications to find spaces of Logarithmic type $L$ such that $L \subset Q_{D}$. As was mentioned before, it was proved, in [2], that the space $L \log L \log \log \log L(\mathbb{T}) \subset Q A$. We shall extend this result to our general context.

Another situation we consider in this work is the following: Let $\Omega$ be any domain in $\mathbb{R}^{n}$, let $W^{1, p}(\Omega)$ be the classical Sobolev space and set $W_{0}^{1, p}(\Omega)$ the closure of $C_{0}^{\infty}(\Omega)$ in $W^{1, p}(\Omega)$, under the norm

$$
\|f\|_{W_{0}^{1, p}(\Omega)}=\|f\|_{p}+\|\nabla f\|_{p},
$$

where $\nabla f$ is the gradient of $f$. Let $T$ be a sublinear operator such that

$$
T: W_{0}^{1, p}(\Omega) \longrightarrow L^{p, \infty}
$$

is bounded with constant $C_{p}$ for every $p \in I \subset[1, \infty)$. Then, for every $f$ such that $\|f\|_{\infty}+\|\nabla f\|_{\infty} \leq 1$, it holds that

$$
(T f)^{*}(t) t^{1 / p} \leq C_{p}\left(\int_{0}^{\infty} f^{*}(t)^{p}+|\nabla f|^{*}(t)^{p} d t\right)^{1 / p} \leq C_{p}\left(\int_{0}^{\infty} f^{*}(t)+|\nabla f|^{*}(t) d t\right)^{1 / p} .
$$

Consequently,

$$
(T f)^{*}(t) \leq \inf _{p \in I}\left(C_{p}\left(\frac{\|f\|_{W_{0}^{1,1}(\Omega)}}{t}\right)^{1 / p}\right):=h\left(t,\|f\|_{W_{0}^{1,1}(\Omega)}\right) .
$$

Then we show that the technique developed in Section 2 can also be extended to cover this situation and, in fact, our theory can be presented in the setting of compatible pairs of Banach spaces $\bar{A}=\left(A_{0}, A_{1}\right)$ using some of the ideas developed in [8]; that is, our operator $T$ will be a sublinear operator acting on elements of the sum space $A_{0}+A_{1}$ and taking values on the set of measurable functions:

$$
T: A_{0}+A_{1} \longrightarrow L^{0}(\mu) .
$$

Our first task is to extend the notion of characteristic functions to the setting of pairs. This will be done in Section 3. 
As usual, the symbol $f \approx g$ will indicate the existence of a universal positive constant $C$ (independent of all parameters involved) so that $(1 / C) f \leq g \leq C f$, while the symbol $f \preceq g$ means that $f \leq C g$. $(\mathcal{M}, \mu)$ will be a totally $\sigma$-finite resonant measure space and we shall denote by $L^{0}(\mu)$ the class of measurable functions that are finite $\mu$ a.e., endowed with the topology of the convergence in measure. We write $\|g\|_{p}$ to denote $\|g\|_{L^{p}(\mu)}$, $\lambda_{g}^{\mu}(y)=\mu(\{x \in \mathcal{M}:|g(x)|>y\})$ is the distribution function of $g$ with respect to the measure $\mu$ and $g_{\mu}^{*}(t)=\inf \left\{s: \lambda_{g}^{\mu}(s) \leq t\right\}$ is the decreasing rearrangement (we refer the reader to [3] for further information about distribution functions and decreasing rearrangements).

In what follows we shall omit the indices $\mu$ whenever it is clear the measure we are working with.

\section{Main results}

First of all, given a positive concave function $D$ such that $D(0+)=0$, we define the space

$$
\Lambda(D)=\left\{f ;\|f\|_{\Lambda(D)}=\int_{0}^{\infty} D\left(\lambda_{f}(y)\right) d y=\int_{0}^{\infty} f^{*}(s) d D(s)\right\} .
$$

Then, we have that the following properties holds:

Lemma 2.1 Given a positive concave function $D$ such that $D(0+)=0$, we have that

$$
\Lambda(D) \subset L^{1}+L^{\infty}
$$

and

$$
Q_{D} \subset \Lambda(D)
$$

with continuous embeddings.

Proof: The first embedding is well known, since $\min (1, s) \preceq D(s)$ and hence $\|f\|_{L^{1}+L^{\infty}}=\int_{0}^{1} f^{*}(s) d s=\int_{0}^{\infty} \min \left(\lambda_{f}(y), 1\right) d y \preceq \int_{0}^{\infty} D\left(\lambda_{f}(y)\right) d y=\|f\|_{\Lambda(D)}$.

For the second embedding, let us observe that if $\|f\|_{\infty} \leq 1$, then

$$
\|f\|_{\Lambda(D)}=\int_{0}^{1} D\left(\lambda_{f}(y)\right) d y \leq D\left(\int_{0}^{1} \lambda_{f}(y) d y\right)=D\left(\|f\|_{1}\right),
$$

and hence, if $f=\sum_{k} e_{k} f_{k}$, with $\left\|f_{k}\right\|_{\infty} \leq 1$, we obtain that

$$
\|f\|_{\Lambda(D)} \leq \sum_{k} e_{k}\left\|f_{k}\right\|_{\Lambda(D)} \leq \sum_{k} e_{k} D\left(\left\|f_{k}\right\|_{1}\right) \leq\|f\|_{Q_{D}} .
$$


Now we are ready to formulate our first main result:

Theorem 2.1 Let $T$ be a sublinear operator such that

$$
T: L^{1}(\mu)+L^{\infty}(\mu) \longrightarrow L^{0}(\mu)
$$

is bounded, and let us assume that, for every $f \in L^{1} \cap L^{\infty}$ with $\|f\|_{\infty} \leq 1$,

$$
(T f)^{*}(t) \leq h\left(t,\|f\|_{1}\right),
$$

for some positive function $h:(0, \infty) \times(0, \infty) \rightarrow(0, \infty)$ such that for every $t>0$, the function $h(t, \cdot)$ is increasing and, for every $s>0, t \cdot h(t, s)$ is also an increasing function in the variable $t$. Then, if $D$ and $R$ are such that

$$
h(t, s) \leq D(s) R(t),
$$

and $D$ is a concave function satisfying $D(0+)=0$, we have that

$$
T: Q_{D} \longrightarrow M(R)
$$

is bounded.

Although no conditions are assumed on $R$, it is clear that since $t \cdot h(t, s)$ is increasing in the variable $t$, we can assume without loss of generality that this condition also holds for $R$.

Proof: Let $f \in Q_{D}$ and let us write $f=\sum_{k} e_{k} f_{k}$ with $\left\|f_{k}\right\|_{\infty} \leq 1$. Then, by the previous lemma, we have that the convergence of the series is in $L^{1}+L^{\infty}$ and therefore, we can conclude that

$$
(T f)^{*}(t) \leq\left(\sum_{k} e_{k} T f_{k}\right)^{*}(t) .
$$

Using now the basic result together with the hypothesis, we obtain that, for every sequence $\left(a_{k}\right)$ of positive numbers such that $\sum_{k} a_{k}=1$,

$$
\begin{aligned}
(T f)^{*}(3 t) & \leq \sum_{k} e_{k}\left(T f_{k}\right)^{*}(t)+\frac{1}{t} \sum_{k} e_{k} \int_{a_{k} t}^{t}\left(T f_{k}\right)^{*}(u) d u \\
& \leq \sum_{k} e_{k} h\left(t,\left\|f_{k}\right\|_{1}\right)+\frac{1}{t} \sum_{k} e_{k} \int_{a_{k} t}^{t} h\left(u,\left\|f_{k}\right\|_{1}\right) d u .
\end{aligned}
$$

And, using the properties of the function $h$, we conclude that

$$
(T f)^{*}(3 t) \leq \sum_{k} e_{k} D\left(\left\|f_{k}\right\|_{1}\right) R(3 t)+R(3 t) \sum_{k} e_{k} D\left(\left\|f_{k}\right\|_{1}\right) \log \frac{1}{a_{k}},
$$

and hence,

$$
\|T f\|_{M(R)}=\sup _{t} \frac{(T f)^{*}(t)}{R(t)} \leq\|f\|_{Q_{D}}
$$


As was mentioned in the introduction, the point now is to analyze the space $Q_{D}$ to make it useful for the applications. To this end, we have to introduce the following logarithmic spaces:

Definition 2.1 Let $\varphi$ be a positive and concave function such that $\varphi\left(0^{+}\right)=0$. (1) The space $L \log |\log L|(\varphi)$ is defined as the set of measurable functions $f$ such that

$$
\|f\|_{L \log |\log L|(\varphi)}:=\int_{0}^{\infty} f^{*}(s)(1+\log (|\log s|+e)) d \varphi(s)<\infty .
$$

(2) The space $L \log \log L(\varphi)$ is defined as the set of measurable functions $f$ such that

$$
\|f\|_{L \log \log L(\varphi)}:=\int_{0}^{\infty} f^{*}(s)\left(1+\log ^{+} \log ^{+} \frac{1}{s}\right) d \varphi(s)<\infty .
$$

(3) The space $L \log \log \log L(\varphi)$ is defined as the set of measurable functions $f$ such that

$$
\|f\|_{L \log \log \log L(\varphi)}:=\int_{0}^{\infty} f^{*}(s)\left(1+\log ^{+} \log ^{+} \log ^{+} \frac{1}{s}\right) d \varphi(s)<\infty .
$$

We also need the two following technical lemmas:

Lemma 2.2 Let $\Phi(s)=s\left(1+\log ^{+} \frac{1}{s}\right)$ and let $f$ be such that $\|f\|_{\Lambda(\varphi)}=1$. Then

$$
\int_{0}^{\infty} \Phi\left(f^{*}(s) \varphi(s)\right) \frac{d \varphi(s)}{\varphi(s)} \approx \int_{0}^{\infty} \Phi\left(s \varphi\left(\lambda_{f}(s)\right)\right) \frac{d s}{s} \preceq\|f\|_{L \log |\log L|(\varphi)} .
$$

Proof: To show the first equivalence, let $H=f^{*} \circ \varphi^{-1}(s)$. Then one has that

$$
\lambda_{H}(s)=\varphi\left(\lambda_{f}(s)\right)
$$

and, by Proposition 4.3 of [21], we have that

$$
\int_{0}^{\infty} \Phi\left(s \lambda_{H}(s)\right) \frac{d s}{s} \approx \int_{0}^{\infty} \Phi(s H(s)) \frac{d s}{s} .
$$

A simple change of variable ends the proof of the first part.

For the second part, let us consider the sets

$$
E_{0}=\left\{s<1: \varphi(s) f^{*}(s)>\left(\log \frac{1}{s}+e\right)^{-2}\right\},
$$

and

$$
E_{1}=\left\{s \geq 1: \varphi(s) f^{*}(s)>(\log s+e)^{-2}\right\}
$$


Then, we can write

$$
\begin{aligned}
& \int_{0}^{\infty} \Phi\left(f^{*}(s) \varphi(s)\right) \frac{d \varphi(s)}{\varphi(s)}= \\
& =\left(\int_{E_{0}}+\int_{(0,1) \backslash E_{0}}+\int_{E_{1}}+\int_{(1, \infty) \backslash E_{1}}\right) \Phi\left(f^{*}(s) \varphi(s)\right) \frac{d \varphi(s)}{\varphi(s)}=I_{1}+I_{2}+I_{3}+I_{4} .
\end{aligned}
$$

Now,

$$
\begin{aligned}
I_{1} & =\int_{E_{0}} f^{*}(s)\left(1+\log ^{+} \frac{1}{f^{*}(s) \varphi(s)}\right) d \varphi(s) \\
& \leq \int_{0}^{1} f^{*}(s)\left(1+2 \log \left(\log \frac{1}{s}+e\right)\right) d \varphi(s) \leq 2\|f\|_{L \log |\log L|(\varphi))} .
\end{aligned}
$$

On the other hand, since $\Phi$ is increasing, $d \varphi(s) \leq(\varphi(s) / s) d s$ and $1=$ $\|f\|_{\Lambda(\varphi)} \leq\|f\|_{L \log |\log L|(\varphi)}$, we obtain that

$$
\begin{aligned}
I_{2} & \leq \int_{0}^{1} \frac{\left(1+2 \log \left(\log \frac{1}{s}+e\right)\right)}{\left(\log \frac{1}{s}+e\right)^{2}} \frac{d \varphi(s)}{\varphi(s)} \leq \int_{0}^{1} \frac{\left(1+2 \log \left(\log \frac{1}{s}+e\right)\right)}{s\left(\log \frac{1}{s}+e\right)^{2}} d s \\
& \preceq\|f\|_{L \log |\log L|(\varphi)} .
\end{aligned}
$$

Similarly,

$$
I_{3} \leq \int_{1}^{\infty} f^{*}(s)(1+2 \log (\log s+e)) d \varphi(s) \preceq\|f\|_{L \log |\log L|(\varphi)},
$$

and

$$
I_{4} \leq \int_{1}^{\infty} \frac{(1+2 \log (\log s+e))}{s(\log s+e)^{2}} d s \preceq\|f\|_{L \log |\log L|(\varphi)}
$$

Lemma 2.3 ([11]) Let $w$ be a positive and measurable function and let $\varphi$ be a positive and concave function such that $\varphi\left(0^{+}\right)=0$. Then

$$
\int_{0}^{\infty} \varphi\left(\lambda_{f}(s)\right) w(s) d s=\int_{0}^{\infty}\left(\int_{0}^{f^{*}(s)} w(t) d t\right) d \varphi(s) .
$$

Theorem 2.2 Let $D$ be any positive and concave function such that $D\left(0^{+}\right)=0$.

Then,

1)

$$
L \log |\log L|(D) \subset Q_{D}
$$

2) If $s \leq D(s)$, then

$$
L \log \log L(D) \subset Q_{D} .
$$

3) If $s \leq D(s)$ and, for every $0 \leq s \leq 1, D\left(s^{2}\right) \preceq s D(s)$, then

$$
L \log \log \log L(D) \subset Q_{D}
$$


Proof: 1) Let $f \in L \log |\log L|(D)$ be such that $\|f\|_{\Lambda(D)}=1$ and let us write

$$
f=\sum_{i \in \mathbb{Z}} 2^{i+1} f_{i}
$$

where $f_{i}=\frac{1}{2^{i+1}} f \chi_{\left\{2^{i}<|f| \leq 2^{i+1}\right\}}$. Then, for every sequence of positive number $\left(a_{i}\right)_{i}$ such that $\sum_{i \in \mathbb{Z}} a_{i}=1$, we have that

$$
\|f\|_{Q_{D}} \preceq \sum_{i \in \mathbb{Z}} 2^{i} D\left(\left\|f_{i}\right\|_{1}\right)\left(1+\log \frac{1}{a_{i}}\right) \leq \sum_{i \in \mathbb{Z}} 2^{i} D\left(\lambda_{f}\left(2^{i}\right)\right)\left(1+\log \frac{1}{a_{i}}\right) .
$$

Taking now

$$
a_{i}=\frac{2^{i} D\left(\lambda_{f}\left(2^{i}\right)\right)}{\sum_{i} 2^{i} D\left(\lambda_{f}\left(2^{i}\right)\right)}
$$

we conclude that

$$
\|f\|_{Q_{D}} \preceq \int_{0}^{\infty} D\left(\lambda_{f}(s)\right)\left(1+\log \frac{1}{s D\left(\lambda_{f}(s)\right)}\right) d s,
$$

and the result now follows by Lemma 2.2 .

2) Since $s \leq D(s)$ we have that $L \log \log L(D) \subseteq \Lambda(D) \subseteq L^{1}$. Let $f \in$ $L \log \log L(D)$ be such that $\|f\|_{\Lambda(D)}=1$, and decompose $f$ as

$$
f=f \chi_{\{|f| \leq 1\}}+\left(\sum_{i \geq 0} 2^{i+1} f_{i}\right)
$$

where $f_{i}=\frac{1}{2^{i+1}} f \chi_{\left\{2^{i}<|f| \leq 2^{i+1}\right\}}$. Then, for every $\left(a_{i}\right)_{i}$ such that $\sum_{i} a_{i}=1$,

$$
\|f\|_{Q_{D}} \preceq D\left(\|f\|_{1}\right)+\sum_{i \geq 0} 2^{i} D\left(\lambda_{f}\left(2^{i}\right)\right)\left(1+\log \frac{1}{a_{i}}\right),
$$

and taking $\left(a_{i}\right)_{i}$ as in 1$)$, we get

$$
\|f\|_{Q_{D}} \preceq 1+\int_{1}^{\infty} D\left(\lambda_{f}(s)\right)\left(1+\log \frac{1}{s D\left(\lambda_{f}(s)\right)}\right) d s \preceq 1+I .
$$

To estimate $I$, it follows, by Lemma 2.2 , that

$$
I \preceq \int_{\left\{f^{*} \geq 1\right\}} f^{*}(s)\left(1+\log \frac{1}{f^{*}(s) D(s)}\right) d D(s),
$$

and since $s \lambda_{f^{*}}(s) \leq 1$, we get that $\lambda_{f^{*}}(s) \leq 1$ if $s \geq 1$. Hence, $\left\{f^{*} \geq 1\right\} \subseteq$ $[0,1]$ and using the same argument than in the proof of Lemma 2.2, it follows that

$$
I \preceq \int_{0}^{1} f^{*}(s)\left(1+\log \frac{1}{f^{*}(s) D(s)}\right) d D(s) \preceq\|f\|_{L \log \log L(D)} .
$$


3) In this case, we take $f \in L \log \log \log L(D)$ such that $\|f\|_{\Lambda(D)}=1$ and we write

$$
f=f \chi_{\{|f| \leq 2\}}+\sum_{i=0}^{\infty} 2^{2^{i+1}} f_{i}
$$

where

$$
f_{i}=\frac{1}{2^{2^{i+1}}} f \chi_{\left\{2^{2^{i}}<|f| \leq 2^{2^{i+1}}\right\}} .
$$

Then, if $\sum_{i} a_{i}=1$,

$$
\begin{aligned}
\|f\|_{Q_{D}} & \preceq 1+\sum_{i=0}^{\infty} 2^{2^{i+1}} D\left(\left\|f_{i}\right\|_{1}\right)\left(1+\log \frac{1}{a_{i}}\right) \\
& \preceq 1+\sum_{i=0}^{\infty} 2^{2^{i+1}} D\left(\frac{1}{2^{2^{i+1}}} \sum_{j=2^{i}}^{2^{i+1}-1} 2^{j} \lambda_{f}\left(2^{j}\right)\right)\left(1+\log \frac{1}{a_{i}}\right),
\end{aligned}
$$

and since $D$ is concave,

$$
\|f\|_{Q_{D}} \preceq 1+\sum_{i=0}^{\infty} 2^{2^{i+1}} \sum_{j=2^{i}}^{2^{i+1}-1} D\left(\frac{2^{j}}{2^{2^{i+1}}} \lambda_{f}\left(2^{j}\right)\right)\left(1+\log \frac{1}{a_{i}}\right) .
$$

Now, using $D(s) / s$ decreases, and that $2^{i} \leq j<2^{i+1}$, we obtain that

$$
2^{2^{i+1}} D\left(\frac{2^{j}}{2^{2^{i+1}}} \lambda_{f}\left(2^{j}\right)\right) \leq\left(2^{j}\right)^{2} D\left(\frac{\lambda_{f}\left(2^{j}\right)}{2^{j}}\right) .
$$

Now we take $a_{i}=6 /\left(\pi^{2}(i+1)^{2}\right)$, and hence

$$
\begin{aligned}
\|f\|_{Q_{D}} & \preceq 1+\sum_{i=0}^{\infty} \sum_{j=2^{i}}^{2^{i+1}-1}\left(2^{j}\right)^{2} D\left(\frac{\lambda_{f}\left(2^{j}\right)}{2^{j}}\right)(1+\log (i+1)) \\
& \preceq \sum_{i=0}^{\infty} \sum_{j=2^{i}}^{2^{i+1}-1}\left(2^{j}\right)^{2} D\left(\frac{\lambda_{f}\left(2^{j}\right)}{2^{j}}\right)\left(1+\log ^{+} \log ^{+} \log ^{+} 2^{j}\right) \\
& \preceq \int_{1}^{\infty} s D\left(\frac{\lambda_{f}(s)}{s}\right)\left(1+\log ^{+} \log ^{+} \log ^{+} s\right) d s .
\end{aligned}
$$

Using that $s \lambda_{f}(s) \leq 1$, we get that

$$
\frac{s}{\lambda_{f}(s)} \leq\left(\frac{1}{\lambda_{f}(s)}\right)^{2}
$$

and since $s D(1 / s)$ increases

$$
s D\left(\frac{\lambda_{f}(s)}{s}\right) \leq \frac{1}{\lambda_{f}(s)} D\left(\left(\lambda_{f}(s)\right)^{2}\right) .
$$


Moreover, since $\lambda_{f}(s) \leq s \lambda_{f}(s) \leq 1$, if $s \geq 1$ and $D\left(s^{t}\right) \preceq s D(s)$,

$$
\frac{1}{\lambda_{f}(s)} D\left(\left(\lambda_{f}(s)\right)^{2}\right) \preceq D\left(\lambda_{f}(s)\right) .
$$

Using this estimate and Lemma 2.3 we get

$$
\begin{aligned}
I & \preceq \int_{0}^{\infty} D\left(\lambda_{f}(s)\right)\left(1+\log ^{+} \log ^{+} \log ^{+} s\right) d s \\
& =\int_{0}^{\infty}\left(\int_{0}^{f^{*}(s)}\left(1+\log ^{+} \log ^{+} \log ^{+} t\right) d t\right) d D(s) .
\end{aligned}
$$

Now, since $\left(1+\log ^{+} \log ^{+} \log ^{+} t\right)$ is increasing and $s f^{*}(s) \leq 1$

$$
\begin{aligned}
I & \leq \int_{0}^{\infty} f^{*}(s)\left(1+\log ^{+} \log ^{+} \log ^{+} f^{*}(s)\right) d D(s) \\
& \leq \int_{0}^{\infty} f^{*}(s)\left(1+\log ^{+} \log ^{+} \log ^{+} \frac{1}{s}\right) d D(s)=\|f\|_{L \log \log \log L(D)} .
\end{aligned}
$$

Let us now define the space $G=\left\{f ;\|f\|_{G}<\infty\right\}$, where

$$
\|f\|_{G}=\inf \left\{\sum_{k=0}^{\infty}\left(c_{k+1}-c_{k}\right) D\left(\frac{\int_{c_{k}}^{c_{k+1}} \lambda_{f}(y) d y}{c_{k+1}-c_{k}}\right) \log (k+2)<\infty\right\},
$$

where the infimum extends over all sequences increasing $\left(c_{k}\right)_{k}$ such that $c_{0}=0$ and $\lim _{k \rightarrow \infty} c_{k}=\infty$.

Proposition 2.1 It holds that $G \subset Q_{D}$.

Proof: Let $f \in G$ and let $\left(c_{k}\right)_{k}$ be a sequence such that

$$
\sum_{k=0}^{\infty}\left(c_{k+1}-c_{k}\right) D\left(\frac{\int_{c_{k}}^{c_{k+1}} \lambda_{f}(y) d y}{c_{k+1}-c_{k}}\right) \log (k+2)<\infty .
$$

Then we write

$$
f=\sum_{k}\left(c_{k+1}-c_{k}\right) f_{k}+\min \left(|f|, c_{1}\right)
$$

where

$$
f_{k}=\frac{\min \left(|f|, c_{k+1}\right)-\min \left(|f|, c_{k}\right)}{c_{k+1}-c_{k}}
$$

and since

$$
\left\|f_{k}\right\|_{1}=\frac{\int_{c_{k}}^{c_{k+1}} \lambda_{f}(y) d y}{c_{k+1}-c_{k}}
$$

we conclude that $f \in Q_{D}$ and $\|f\|_{Q_{D}} \preceq\|f\|_{G}$. 
Remark 2.1 By taking $c_{k}=2^{2^{k}}$, one can easily see that under the conditions of Theorem 2.2, 3), we have that $L \log \log \log L(D) \subset G$.

If $T$ is the Carleson maximal operator $S$, then one can immediately see that we can take $D(s)=s\left(1+\log ^{+} \frac{1}{s}\right)$ and $R(t)=t$. In this particular case, the above result has been recently obtained by Arias-de-Reyna in [2]. Also, for such function $D$, it is very easy to see that

$$
L \log \log \log L(D)=L \log L \log \log \log L(\mathbb{T}),
$$

and the boundedness

$$
S: L \log L \log \log \log L(\mathbb{T}) \longrightarrow L^{1, \infty},
$$

was obtained previously by Antonov in [1], and for other more general operators, as mentioned in the introduction, in [19].

\section{Extension to arbitrary compatible pairs}

Let $\bar{A}=\left(A_{0}, A_{1}\right)$ be a compatible pair of Banach spaces, that is, we assume that there is a topological vector space $\mathcal{U}$ such that $A_{i} \subset \mathcal{U}, i=0,1$, continuously. In what follows we drop the terms "compatible" and "Banach" and refer to a compatible Banach pair simply as a "pair".

The Peetre $K$-functional (see [3], [4] and [5]) associated with a pair $\bar{A}$ is defined, for each $a \in A_{0}+A_{1}$ and $t>0$, by

$$
K(a, t)=K(a, t ; \bar{A})=\inf \left\{\left\|a_{0}\right\|_{A_{0}}+t\left\|a_{1}\right\|_{A_{1}}: a=a_{0}+a_{1}, a_{i} \in A_{i}\right\} .
$$

It is easy to see that $K(t, a)$ is a nonnegative and concave function of $t>0$, (and thus also continuous). Therefore

$$
K(a, t ; \bar{A})=K\left(a, 0^{+} ; \bar{A}\right)+\int_{0}^{t} k(a, s ; \bar{A}) d s,
$$

where the $k$-functional, $k(a, s ; \bar{A})=k(a, s)$, is a uniquely defined, nonnegative, decreasing and right-continuous function of $s>0$.

In order to find the analogue of the set $\left\{f \in L^{1} ;\|f\|_{\infty} \leq 1\right\}$ in the setting of pairs, let us recall that the Gagliardo completion $\tilde{A}_{0}$ and $\tilde{A}_{1}$ of a pair $\bar{A}$ is defined by (see [3])

$$
\begin{aligned}
\|a\|_{\tilde{A}_{0}} & =\sup _{t} K(t, a ; \bar{A})<\infty, \\
\|a\|_{\tilde{A}_{1}} & =\sup _{t} \frac{K(t, a ; \bar{A})}{t}<\infty .
\end{aligned}
$$


Definition 3.1 Given a pair $\bar{A}$, we say that a is a characteristic element of $\bar{A}$ if $a \in \tilde{A}_{0} \cap \tilde{A}_{1}$ and $\|a\|_{\tilde{A}_{1}} \leq 1$.

The collection of characteristic elements of a pair $\bar{A}$ will be denoted by $C(\bar{A})$.

The following lemma was proved in [8] and it is fundamental for our purpose.

Lemma 3.1 Given an element $a \in A_{0}+A_{1}$ such that $K\left(a, 0^{+} ; \bar{A}\right)=0$, there exist a constant $\gamma$ (depending only on $\bar{A}$ ) and a collection of characteristic elements $\left(a_{i}\right)_{i \in \mathbb{Z}}$ such that

$$
a=\gamma \sum_{i \in \mathbb{Z}} 2^{i} a_{i}\left(\text { convergence in } A_{0}+A_{1}\right)
$$

and

$$
\left\|a_{i}\right\|_{\tilde{A}_{0}} \leq \lambda_{k(a, \cdot)}\left(2^{i}\right)
$$

We say that $a=\gamma \sum_{i=-\infty}^{\infty} 2^{i} a_{i}$ is a dyadic decomposition of $a$.

Definition $3.2([8])$ Given a pair $\bar{A}=\left(A_{0}, A_{1}\right)$ and a concave function $\varphi$, the minimal Lorentz space, $\Lambda(\varphi ; \bar{A})$, is the set of elements $a \in A_{0}+A_{1}$ such that $K\left(a, 0^{+} ; \bar{A}\right)=0$ and

$$
\|a\|_{\Lambda(\varphi ; \bar{A})}=\int_{0}^{\infty} k(a, s ; \bar{A}) d \varphi(s)<\infty
$$

If $\bar{A}$ is the classical pair $\left(L^{1}(\nu), L^{\infty}(\nu)\right)$, then $k(a, s)=f^{*}(s)$ and hence $\Lambda(\varphi ; \bar{A})=\Lambda(\varphi)$ is the classical Lorentz spaces defined in the previous section.

Definition 3.3 Given a pair $\bar{A}$, and a quasi-Banach lattice $B \subset \Lambda(\varphi)$, we define $B(\varphi ; \bar{A})$ as

$$
B(\varphi ; \bar{A})=\left\{a \in \Lambda(\varphi ; \bar{A}) ;\|a\|_{B(\varphi ; \bar{A})}:=\|k(a, \cdot)\|_{B}<\infty\right\} .
$$

Remark 3.1 Obviously,

$$
L \log |\log L|(\varphi ; \bar{A}) \subset L \log \log L(\varphi ; \bar{A}) \subset L \log \log \log L(\varphi ; \bar{A})
$$

and the above embeddings are, in general, strict. However, if $\bar{A}$ is an ordered pair, that is $\left.A_{1} \subset A_{0}\right)$ then $k(a, t)=0$ ift $>1$, and hence $L \log |\log L|(\varphi ; \bar{A})=$ $L \log \log L(\varphi ; \bar{A})$. 
Definition 3.4 Let $h:(0, \infty) \times(0, \infty) \rightarrow(0, \infty)$ be such that for every $t>0$, the function $h(t, \cdot)$ is increasing and, for every $s>0, t \cdot h(t, s)$ is also an increasing function in the variable $t$. We say that a sublinear continuous operator

$$
T: A_{0}+A_{1} \longrightarrow L^{0}(\mu)
$$

satisfies a restricted $h$ - rearrangement inequality if, for every $t>0$ and every characteristic element a of $\bar{A}$,

$$
(T a)^{*}(t) \leq h\left(t,\|a\|_{\tilde{A}_{0}}\right) .
$$

\section{Examples:}

1) If $\bar{A}=\left(L^{1}(\nu), L^{\infty}(\nu)\right)$, then $C(\bar{A})=\left\{f \in L^{1} ;\|f\|_{\infty} \leq 1\right\}$, and hence, any sublinear operator satisfying (3.2), satisfies the condition assumed in the previous section.

2) Let $\Omega$ be any domain in $\mathbb{R}^{n}$ and let $W^{1, p}(\Omega)$ be the classical Sobolev space

$$
\|f\|_{W^{1, p}(\Omega)}=\|f\|_{p}+\|\nabla f\|_{p}
$$

where $\nabla f$ is the gradient of $f$. Set $W_{0}^{1, p}(\Omega)$ the closure of $C_{0}^{\infty}(\Omega)$ in $W^{1, p}(\Omega)$. Then it is known, (see [12]), that if $\bar{A}=\left(W_{0}^{1,1}(\Omega), W_{0}^{1, \infty}(\Omega)\right)$,

$$
K(t, f ; \bar{A}) \approx t\left(f^{* *}(t)+|\nabla f|^{* *}(t)\right),
$$

and therefore $C(\bar{A})=\left\{f \in W_{0}^{1,1}(\Omega) ;\|f\|_{\infty}+\|\nabla f\|_{\infty} \leq 1\right\}$. Hence, if $T$ is a sublinear operator such that

$$
T: W_{0}^{1, p}(\Omega) \longrightarrow L^{p, \infty}
$$

is bounded with constant $C_{p}$ for every $p \in I \subset[1, \infty)$, then,

$$
(T f)^{*}(t) \leq \inf _{p \in I}\left(C_{p}\left(\frac{\|f\|_{W_{0}^{1,1}(\Omega)}}{t}\right)^{1 / p}\right):=h\left(t,\|f\|_{W_{0}^{1,1}(\Omega)}\right) .
$$

3) Let us now consider, for example, the pair $\bar{A}=\left(\Lambda^{1}(w), L^{\infty}\right)$, where $\Lambda^{1}(w)$ is the weighted Lorentz space introduced by Lorentz in [15] and defined by

$$
\|f\|_{\Lambda^{p}(w)}=\left(\int_{0}^{\infty} f^{*}(t)^{p} w(t) d t\right)^{1 / p}<\infty
$$

Let us recall that the weak type version of these spaces are defined by

$$
\|f\|_{\Lambda^{p, \infty}(W)}=\sup _{t>0} f^{*}(t) W^{1 / p}(t)<\infty .
$$


Consider a sublinear operator $T$ such that, for some weights $w$ and $W$,

$$
T: \Lambda^{p}(w) \longrightarrow \Lambda^{p, \infty}(W),
$$

with constant less than or equal to $C_{p}$. Then, since it is known that $K(t, f ; \bar{A})=\int_{0}^{t}\left(f^{*}\right)_{w}^{*}(s) d s$, we can conclude that

$$
C(\bar{A})=\left\{f \in \Lambda^{1}(w):\|f\|_{\infty} \leq 1\right\},
$$

and therefore, for every characteristic element,

$$
(T f)^{*}(t) \leq \inf _{p}\left(C_{p}\left(\frac{\|f\|_{\Lambda^{1}(w)}}{W(t)}\right)^{1 / p}\right):=h\left(t,\|f\|_{\Lambda^{1}(w)}\right) .
$$

Let us now define the space

$$
Q_{D}(\bar{A})=\left\{a=\sum_{k} e_{k} a_{k} ;\left\|a_{k}\right\|_{\tilde{A}_{1}} \leq 1,\|a\|_{Q_{D}(\bar{A})}<\infty\right\},
$$

where

$$
\begin{aligned}
& \|a\|_{Q_{D}(\bar{A})} \\
& =\inf \left\{\sum_{k} e_{k} D\left(\left\|a_{k}\right\|_{\tilde{A}_{0}}\right)\left(1+\log \frac{1}{c_{k}}\right) ; \sum_{k} c_{k}=1, c_{k} \geq 0, a=\sum_{k} e_{k} a_{k}\right\} .
\end{aligned}
$$

Then, we have the following extension of Theorem 2.1:

Theorem 3.1 Let $T: A_{0}+A_{1} \rightarrow L^{0}(\mu)$ be a sublinear operator satisfying a restricted $h$-rearrangement inequality. Then, if $D$ and $R$ are two positive functions such that $D$ is concave, $D\left(0^{+}\right)=0$ and

$$
h(t, s) \leq D(s) R(t)
$$

we have that

$$
T: Q_{D}(\bar{A}) \longrightarrow M(R)
$$

is bounded.

Proof: Given $a \in \Lambda(D ; \bar{A})$ such that $\|a\|_{\Lambda(D ; \bar{A})}=1$, we can decompose $a$ as in Lemma 3.1

$$
a=\gamma \sum_{i \in Z} 2^{i} a_{i}
$$

Then, if $a_{N}=\gamma \sum_{i=-N}^{N} 2^{i} a_{i}$, we have that $T a_{N} \rightarrow T a$ in measure, and therefore,

$$
\left(T a_{N}\right)^{*}(t) \rightarrow(T a)^{*}(t) \quad \text { a.e. } t>0
$$


By the sublinearity of $T$ we get that

$$
\left(T a_{N}\right)^{*}(t) \leq \gamma\left(\sum_{i=-N}^{N} 2^{i}\left|T a_{i}\right|\right)^{*}(t) \leq \gamma\left(\sum_{i=-\infty}^{\infty} 2^{i}\left|T a_{i}\right|\right)^{*}(t)
$$

and hence

$$
(T a)^{*}(t) \leq \gamma\left(\sum_{i=-\infty}^{\infty} 2^{i}\left|T a_{i}\right|\right)^{*}(t) \text { a.e. } t>0
$$

The proof now follows as in Theorem 2.1.

We also have and analogue to Theorem 2.2:

Theorem 3.2 Let $D$ be any positive and concave function $D$ such that $D\left(0^{+}\right)=0$. Then,

$$
L \log |\log L|(D ; \bar{A}) \subset Q_{D}(\bar{A}) .
$$

2) If $s \leq D(s)$, then

$$
L \log \log L(D ; \bar{A}) \subset Q_{D}(\bar{A}) .
$$

3) $s \leq D(s)$ and, for every $0 \leq s \leq 1, D\left(s^{2}\right) \preceq s D(s)$, then

$$
L \log \log \log L(D ; \bar{A}) \subset Q_{D}(\bar{A}) .
$$

Proof: 1) In this case, given $a \in \Lambda(D ; \bar{A})$ such that $\|a\|_{\Lambda(D ; \bar{A})}=1$, we decompose $a$ as in Lemma 3.1

$$
a=\gamma \sum_{i \in Z} 2^{i} a_{i}
$$

and continue as in the proof of Theorem 2.2, 1).

2) Since $s \leq D(s)$ we have that $L \log \log L(D ; \bar{A}) \subseteq \Lambda(D ; \bar{A}) \subseteq \tilde{A}_{0}$. Let $a \in \Lambda(D ; \bar{A})$ such that $\|a\|_{\Lambda(D ; \bar{A})}=1$, and decompose $a$ as

$$
a=\gamma\left(\sum_{i<0} 2^{i} a_{i}+\sum_{i \geq 0} 2^{i} a_{i}\right)=\gamma\left(a^{0}+\sum_{i \geq 0} 2^{i} a_{i}\right) .
$$

Then since $a^{0} \in C(\bar{A})$, and $\left\|a_{i}\right\|_{\tilde{A}_{0}} \leq \lambda_{k(a, \cdot)}\left(2^{i}\right)$, we have that

$$
\begin{aligned}
\|a\|_{Q_{D}(\bar{A})} & \preceq D\left(\left\|a^{0}\right\|_{\tilde{A}_{0}}\right)+\int_{1}^{\infty} D\left(\lambda_{k(a, \cdot)}(s)\right)\left(1+\log \frac{1}{s D\left(\lambda_{k(a, \cdot)}(s)\right)}\right) d s \\
& =I_{1}+I_{2} .
\end{aligned}
$$

Obviously

$$
I_{1} \preceq D\left(\|a\|_{\tilde{A}_{0}}\right) \leq D\left(\|a\|_{\Lambda(D ; \bar{A})}\right)=D(1) \leq D(1)\|a\|_{L \log \log L(D ; \bar{A})},
$$

and to estimate $I_{2}$, we follow as in the proof of Theorem 2.2,2). 
3) In this case, given $a \in L \log \log \log L(D ; \bar{A})$ such that $\|a\|_{\Lambda(D ; \bar{A})}=1$, let $a=\gamma \sum_{i \in \mathbb{Z}} 2^{i} a_{i}$ be a dyadic decomposition. Then, if, for every $k \in \mathbb{N}$, $d_{k}=\sum_{i=2^{k}}^{2^{k+1}-1} 2^{i}$, we obtain that

$$
a=\sum_{i=-\infty}^{0} 2^{i} a_{i}+\sum_{k=0}^{\infty} d_{k}\left(\frac{1}{d_{k}} \sum_{i=2^{k}}^{2^{k+1}-1} 2^{i} a_{i}\right)=a^{0}+\sum_{k=0}^{\infty} d_{k} A_{k}
$$

where, it is immediate to see that both $a^{0}$ and $A_{k}$ are characteristic elements. Then, for every $\sum_{k} c_{k}=1$,

$$
\|a\|_{Q_{D}(\bar{A})} \leq D\left(\|a\|_{\tilde{A}_{0}}\right)+\sum_{k=0}^{\infty} d_{k} D\left(\left\|A_{k}\right\|_{\tilde{A}_{0}}\right)\left(1+\log \frac{1}{c_{k}}\right)=\left(D\left(\|a\|_{\tilde{A}_{0}}\right)+I\right) .
$$

Since $a_{i} \in C(\bar{A})$ and $D$ is subadditive, we have that

$$
d_{k} D\left(\left\|A_{k}\right\|_{\tilde{A}_{0}}\right) \leq d_{k} \sum_{i=2^{k}}^{2^{k+1}-1} D\left(\frac{2^{i}}{d_{k}}\left\|a_{i}\right\|_{\tilde{A}_{0}}\right) \leq d_{k} \sum_{i=2^{k}}^{2^{k+1}-1} D\left(\frac{2^{i}}{d_{k}} \lambda_{k(a, \cdot)}\left(2^{i}\right)\right)
$$

and the proof now follows as in Theorem 2.2,3).

\section{Applications}

Let $T$ be a sublinear operator satisfying a restricted $h$-rearrangement inequality, where

$$
h(t, s)=\frac{s}{t}\left(1+\log ^{+} \frac{t}{s}\right)^{m}
$$

with $m>0$, as it happens with the examples we have mentioned in the introduction. Then,

$$
h(t, s) \leq \frac{s}{t}\left(1+\log ^{+} \frac{t}{s}\right)^{m} \leq s\left(1+\log ^{+} \frac{1}{s}\right)^{m} \frac{1}{t}\left(1+\log ^{+} t\right)^{m},
$$

and we can take $D(s)=s\left(1+\log ^{+} \frac{1}{s}\right)^{m}$ and $R(t)=\frac{1}{t}\left(1+\log ^{+} t\right)^{m}$ in our Theorems 3.1 and 3.2 to conclude the following result.

Theorem 4.1 If $T: A_{0}+A_{1} \rightarrow L^{0}(\mu)$ satisfies a restricted $h$-rearrangement inequality with $h(t, s)=\frac{s}{t}\left(1+\log ^{+} \frac{t}{s}\right)^{m}, T$ can be extended continuously

$$
T: Q_{D}(\bar{A}) \longrightarrow M(R),
$$

where $D(t)=t\left(1+\log ^{+} \frac{1}{t}\right)^{m}$, and $R(t)=\frac{1}{t}\left(1+\log ^{+} t\right)^{m}$.

In particular, $T: L \log \log \log L(D ; \bar{A}) \longrightarrow M(R)$ is bounded, where

$L \log \log \log L(D ; \bar{A})=\left\{a \in A_{0}+A_{1} ; k(a, \cdot) \in L(\log L)^{m} \log \log \log L\right\}$,

with $\|a\|_{L \log \log \log L(D ; \bar{A})}=\|k(a, \cdot)\|_{L(\log L)^{m} \log \log \log L}$. 


\section{Examples}

(I) If $\bar{A}=\left(L^{1}(\mathbb{T}), L^{\infty}(\mathbb{T})\right), k(f, \cdot)=f^{*}$ and we recover the result of Section 2 .

(II) If $\bar{A}=\left(W_{0}^{1,1}(\Omega), W_{0}^{1, \infty}(\Omega)\right)$ where $\Omega$ has finite measure, and

$$
T: W_{0}^{1, p}(\Omega) \longrightarrow L^{p, \infty}
$$

is bounded with constant say $1 /(p-1)$, then, applying Theorem 4.1 , we obtain that

$$
T: W_{0}(\Omega) \longrightarrow L^{1, \infty}
$$

is bounded, where $W_{0}(\Omega)$ is the closure of $C_{0}^{\infty}(\Omega)$ in $W(\Omega)$ with

$$
W(\Omega)=\{f ;|f|+|\nabla f| \in L \log L \log \log \log L\} .
$$

(III) In all our previous applications we have considered sublinear operators with values in $L^{p, \infty}$ where the constant blows up when $p$ tends to 1 . This was the unique interested case since if $p$ tend to $p_{0}$ with $p_{0} \neq 1$, then we can substitute $(T f)^{*}$ by $(T f)^{* *}$ without a change in the behaviour of the constant and hence we can apply the strong type extrapolation theory studied in [7] and [8] instead of the theory developed in this work to obtain better results. However this is not the general case.

Our third application deals with the theory of weighted Lorentz spaces and with a sublinear operator $T$ with values in spaces of the form $\Lambda^{p, \infty}\left(W_{1}\right)$ where $f^{*}$ can not be, in general, substituted by $f^{* *}$ even if $p \neq 1$. Let $w_{0}$ and $W_{1}$ be weights in $(0, \infty)$ and let $T$ be a sublinear operator such that

$$
T: \Lambda^{1}\left(w_{0}\right)+L^{\infty} \longrightarrow L^{0}\left(\mathbb{R}^{n}\right)
$$

is continuous and, for every $p>2$,

$$
T: \Lambda^{p}\left(w_{0}\right) \longrightarrow \Lambda^{p, \infty}\left(W_{1}\right)
$$

is bounded with constant $p$ (see, [16], [17], [10] to find examples of operators $T$ satisfying the above condition); that is

$$
(T f)^{*}(t) W_{1}(t)^{1 / p} \leq p\left(\int_{0}^{\infty} f^{*}(s) w_{0}(s) d s\right)^{1 / p} .
$$

Now, if we take $\bar{A}=\left(\Lambda^{1}\left(w_{0}\right), L^{\infty}\right)$, we have that $\tilde{A}_{0}=\Lambda^{1}\left(w_{0}\right)$ and hence, it follows, taking the infimum in $p>2$, that

$$
(T f)^{*}(t) \preceq h\left(W_{1}(t),\|a\|_{\tilde{A}_{0}}\right),
$$

where $h(t, s)=\inf _{p>2} p(s / t)^{1 / p} \approx(s / t)^{1 / 2}\left(1+\log ^{+}(s / t)\right)$. Therefore, we can deduced the following result. 
Theorem 4.2 Let $T$ be a sublinear operator as above. Then, $T$ can be extended continuously

$$
T: L \log |\log L|(D ; \bar{A}) \rightarrow M(R)
$$

where $D(s)=s^{1 / 2}\left(1+\log ^{+} s\right)$, and $R(t)=W_{1}(t)^{-1 / 2}\left(1+\log ^{+} \frac{1}{W_{1}(t)}\right)^{-1}$.

Open Question: When is is true that the space $L \log \log \log \log L(D ; A) \subset$ $Q_{D}(\bar{A})$ or in general, $L \log ^{(m)} L(D ; A) \subset Q_{D}(\bar{A})$ ?

\section{References}

[1] Antonov, N. Y.: Convergence of Fourier Series. East J. Approx. 2 (1996), no. $2,187-196$.

[2] Arias-de-Reyna, J.: Pointwise Convergence of Fourier Series. J. London Math. Soc. (2) 65 (2002), no. 1, 139-153.

[3] Bennett, C. and Sharpley, R.: Interpolation of Operators. Academic Press, Boston, 1988.

[4] Bergh, J. and Lofström, J.: Interpolation spaces. An introduction. Springer, New York, 1976.

[5] Brudnyi, Yu. A. And Krugluak, N. Ya.: Interpolation Functors and Interpolation Spaces. North-Holland, Amsterdam, 1991.

[6] Carleson, L.: On convergence and growth of partial sums of Fourier series. Acta Math. 116 (1966), 135-157.

[7] Carro, M. J.: New extrapolation estimates. J. Funct. Anal. 174 (2000), no. $1,155-166$.

[8] Carro, M. J. and Martín, J.: An abstract extrapolation theory for the real interpolation method. Collect. Math. 53 (2002), no. 2, 165-186.

[9] Carro, M. J. and Martín, J.: A useful estimate for the decreasing rearrangement of a sum of functions. To appear in Quart. J. Math.

[10] Carro, M. J., Raposo, J. A. and Soria, J.: Recent developments in the theory of Lorentz spaces and weighted inequalities. Preprint (2001).

[11] Carro, M. J. And Soria, J.: Weighted Lorentz spaces and the Hardy operator. J. Funct. Anal. 112 (1993), 480-494.

[12] DeVore, R. And Scherer, K.: Interpolation of linear operators on Sobolev spaces. Ann. of Math. 109 (1979), 583-609.

[13] Hunt, R. A.: On the convergence of Fourier series. In Orthogonal Expansions and their Continuous Analogues (Proc. Conf., Edwardsville, Ill., 1967), 235-255. Southern Illinois Univ. Press, Carbondale, Ill., 1967.

[14] Jawerth, B. And Milman, M.: Extrapolation Theory with Applications. Mem. Amer. Math. Soc. 89 (1991), no. 440. 
[15] Lorentz, G. G.: On the theory of spaces $\Lambda$. Pacific J. Math. 1 (1951), $411-429$.

[16] Muckenhoupt, B.: Weighted norm inequalities for the Hardy-Littlewood maximal function. Trans. Amer. Math. Soc. 165 (1972), 207-226.

[17] SAwyer, E.: Boundedness of classical operators on classical Lorentz spaces. Studia Math. 96 (1990), 145-158.

[18] SjÖLIn, P.: An inequality of Paley and convergence a.e. of Walsh-Fourier series. Ark. Mat. 7 (1969), 551-570.

[19] SuÖlin, P. And Soria, F.: Remarks on a theorem by N. Yu. Antonov. Studia Math. 158 (2003), no. 1, 79-97.

[20] Soria, F.: On an extrapolation theorem of Carleson-Sjölin with applications to a.e. convergence of Fourier series. Studia Math. 94 (1989), 235-244.

[21] SoriA, F.: Characterizations of classes of functions generated by blocks and associated Hardy spaces. Indiana Univ. Math. J. 34 (1985), 463-492.

[22] Yano, S.: Notes on Fourier analysis. XXIX. An extrapolation theorem. J. Math. Soc. Japan 3 (1951), 296-305.

Recibido: 11 de abril de 2002

María J. Carro

Departament de Matemàtica Aplicada i Anàlisi

Universitat de Barcelona

E-08071 Barcelona, Spain

carro@mat.ub.es

Joaquim Martín

Departament de Matemàtiques

Universitat Autònoma de Barcelona Bellaterra, E-08193, Barcelona, Spain

jmartin@mat.uab.es

This work has been partially supported by the CICYT BFM2001-3395 and by CURE 2001SGR 00069. The second author has also been supported by "Programa Ramón y Cajal", MCYT. 